\title{
Conservação do farelo de arroz integral por ensilagem com diferentes graus de hidratação e adição de fubá de milho
}

\section{Conservation of the rice bran for silage with different levels of hydration and addition of cornmeal}

\author{
TUBIN, Jiovani Sergio Bee ${ }^{1}$; CONTE, Renato Augusto ${ }^{1}$; BAGGIO, Rafael Alan ${ }^{1}$; DAL \\ PIVO, João Carlos ${ }^{1}$; ZANELLA, Mariângela ${ }^{1}$; PAIANO, Diovani ${ }^{1 *}$
}

\author{
${ }^{1}$ Universidade do Estado de Santa Catarina, Centro de Educação Superior do Oeste, Departamento de \\ Zootecnia, Chapecó, Santa Catarina, Brasil. \\ *Endereço para correspondência: diovani@hotmail.com
}

\section{RESUMO}

Foi conduzido um estudo para avaliar diferentes graus de hidratação e adição de fubá de milho (FMI) para a ensilagem do farelo de arroz integral (FAR) para conservação do produto. Foram utilizadas relações FAR: FMI 10:0; 9:1; 8:2; 7:3 e 6:4 e níveis de hidratação da mistura $10,20,30,40$ e $50 \%$ do peso inicial, perfazendo um esquema fatorial de $5 \times 5$ com quatro repetições. Paralelamente, foram fabricados quatro silos de características semelhantes aos demais com FAR sem a hidratação, denominados silos referência, e avaliada a peroxidação lipídica. Após a abertura (120 dias de ensilagem) foram avaliados $\mathrm{o} \mathrm{pH}$, acidez titulável (AT), índice de acidez (IA), índice de fungos (IF) e atividade de água (Aw). A temperatura (análise para verificar a estabilidade aeróbica) e o $\mathrm{pH}$, pós abertura, foram determinados em 24, 48 e 120 horas após a abertura. Houve interações entre as variáveis para: $\mathrm{pH}$ na abertura, AT, IA e IF. Para a variável Aw não houve interação. A inclusão de $40 \%$ de FMI e 42,3\% de água melhorou o pH da silagem na abertura, e a inclusão de $43,6 \%$ de água melhorou o pH 24 horas pós abertura. Após 48 horas de abertura as silagens estavam inapropriadas para o consumo, e a inclusão do milho acelerou a deterioração da massa ensilada após este período. Somente nos silos controles foi observado peroxidação lipídica. O uso do método de ensilagem para preservar a qualidade do FAR foi promissor como ferramenta para diminuir a peroxidação, independentemente do nível de hidratação. Para a confecção de silagem de farelo de arroz recomenda-se a inclusão de FMI de $40 \%$ e hidratação de $42,3 \%$ sobre o peso para otimizar o $\mathrm{pH}$ na abertura.

Palavras-chave: alimento alternativo, estabilidade aeróbica, peroxidação, rancidez oxidativa

\section{SUMMARY}

A study was carried out to evaluate the addition of cornmeal (FMI) at different hydration degrees to rice bran, as a strategy for its conservation by silage (FAR). Five relations of FAR and FMI were used: $10: 0 ; 9: 1 ; 8: 2 ; 7: 3$ and $6: 4$, combined with five hydration levels: 10, 20, 30, 40 and 50\% over initial weight, performing a $5 \times 5$ factorial design with four replications. In parallel, were prepared four similar silos only with FAR, without hydration (control silos). After 120 days of storage, the $\mathrm{pH}$, titratable acidity, acidity index, fungi index, and water activity (Aw) were evaluated. The temperature (regarding to verify the aerobic stability) and the $\mathrm{pH}$ were determined at 24, 48 and 120 hour after opening. Interactions were observed for $\mathrm{pH}$ at opening time, titratable acidity, acid index and fungi index. No interactions were observed with Aw. The inclusion of $40 \%$ of cornmeal and $42.3 \%$ water improved the silage $\mathrm{pH}$ at opening and 43,6\% improve the silage $\mathrm{pH}$ at 24 hours after opening. However, after $48 \mathrm{~h}$ of opening, all silages were inappropriate for consumption, the addition of cornmeal accelerated the deterioration of silage. Only on the control silos, the peroxidation process was observed. Then, the use of silage method for preserving the quality of rice bran was promising as a tool to reduce peroxidation independent of hydration level. We recommended for silage rice bran the inclusion of FMI $40 \%$ and $42.3 \%$ of water to optimize the $\mathrm{pH}$ in the opening.

Keywords: aerobic stability, alternative food, oxidative rancidity, peroxidation 


\section{INTRODUÇÃO}

O farelo de arroz integral (FAR) é coproduto do beneficiamento ou polimento do arroz, constituído de pericarpo, testa, aleurona, gérmen $\mathrm{e}$ amido, dependentes do grau de polimento (KAUFMANN et al., 2005). Na safra 2015 o Brasil produziu 12,5 milhões de toneladas de arroz (CONAB, 2015) e o beneficiamento produz em média $8 \%$ de FAR (ÁVILA et al., 2003).

Uma característica relevante do FAR é o alto teor de óleo, entre 14,5 a 21,4\%, de proteína bruta (PB), que varia de 8,0 a 17\%, (ALI et al., 1998; ROSTAGNO et al., 2011) e grande quantidade de enzimas lipolíticas (MUJAHID et al., 2003).

Após a moagem, as enzimas lipolíticas são ativadas, o que resulta em rápida hidrólise dos triglicerídeos e ocasiona rancificação dos ácidos graxos (SAUNDERS, 1990), com incremento na acidez de até $1 \%$ por hora (CORDERO et al., 1985).

Com vistas à conservação e preservação da qualidade do FAR a ensilagem pode ser uma alternativa, pois utiliza das mesmas tecnologias da ensilagem de grão reidratados de milho, cuja prática segundo Pereira et al. (2013) é simples e barata. A silagem é um método de conservação, sob condições anaeróbicas, pelo qual as bactérias láticas convertem hidratos de carbono solúveis em água e em ácidos orgânicos, como resultado o $\mathrm{pH}$ diminui e a silagem é preservada por longos períodos se não exposta ao ambiente aeróbico (WEINBERG \& CHEN, 2013).

$\mathrm{O}$ fato da ensilagem ocorrer em ambiente anaeróbico poderia ser uma alternativa para restrição do processo de rancidez oxidativa.

Uma das limitações do processo é que na abertura do silo há aeração da massa o que permite a ação de leveduras que oxidam os ácidos orgânicos conservantes da silagem, que desencadeia a degradação aeróbia e a elevação do pH (CASTRO et al., 2006), com comprometimento da qualidade do material ensilado.

Contudo, o elevado nível de PB do FAR pode dificultar a acidificação da massa pela maior capacidade tampão. Para minimizar esta dificuldade o fubá de milho (FMI) poderia ser uma alternativa como melhorador da ensilagem pelo seu maior teor de carboidratos solúveis. Entretanto, é escassa a literatura que trata da conservação do FAR pela ensilagem com a utilização do FMI como aditivo.

Abordoado no exposto, susceptibilidade à degradação, redução da rancificação, baixo custo e escassa literatura, foi conduzido um estudo com os objetivos de avaliar o processo de ensilagem do farelo de arroz com diferentes graus de hidratação e adição de FMI, sobre a qualidade e a estabilidade aeróbica.

\section{MATERIAL E MÉTODOS}

Para o estudo foi utilizado um lote de farelo de arroz integral (FAR) com cinco dias de processamento e um lote de milho, os quais foram obtidos no comércio regional de Chapecó-SC. A composição química do FAR e do FMI (Tabela 1), foram obtidas conforme metodologia preconizada por Silva \& Queiroz (2002). Foram fabricados mini silos com $2 \mathrm{~kg}$ de matéria natural, volume que permitiu avaliar adequadamente, após a abertura, a estabilidade aeróbica. Optouse pela utilização de mini silos pois estudos de Neumann et al. (2007), não encontrarem diferenças para o $\mathrm{pH}$ de silagens de planta de milho entre o silo tipo trincheira e silos laboratoriais.

Os mini-silos foram confeccionados com sacos plásticos de alta densidade de cinco litros, fechados hermeticamente (sob vácuo) e armazenados em ambiente 
Rev. Bras. Saúde Prod. Anim., Salvador, v.17, n.4, p.608-616 out./dez., 2016 http://www.rbspa.ufba.br ISSN 15199940

escuro com temperatura controlada $\left(22^{\circ} \mathrm{C}\right)$, após a ensilagem o gás produzido foi retirado por meio de bomba de vácuo e novamente selados hermeticamente, esta técnica foi repetida diariamente até o encerramento da produção de gases, cerca de 48 horas após a ensilagem não foi mais necessária. A campo o procedimento não se faz necessário pois o fechamento dos silos não será hermético, como no caso dos silos laboratoriais, o que permite o escape de gases produzidos no processo fermentativo.

Tabela 1. Composição química do farelo de arroz integral (FAR) e do fubá de milho (FMI) na matéria natural

\begin{tabular}{|c|c|c|}
\hline Variáveis & Farelo de arroz & Fubá de milho \\
\hline Matéria Seca, $\%^{1}$ & 89,62 & 86,53 \\
\hline Proteína Bruta, $\%^{1}$ & 17,68 & 8,98 \\
\hline Cinzas, $\%^{1}$ & 8,24 & 1,12 \\
\hline Fibra em Detergente Neutro, $\%^{1}$ & 21,52 & 9,49 \\
\hline Fibra em Detergente Ácido, $\%^{1}$ & 13,90 & 3,42 \\
\hline Extrato Etéreo, $\%{ }^{1}$ & 22,01 & 4,33 \\
\hline Energia Bruta, Mcal $/ \mathrm{kg}$ & 5,27 & 4,41 \\
\hline pH Inicial ${ }^{1}$ & 6,26 & 5,30 \\
\hline Capacidade Tampão, $\mathrm{NaOH} / 100 \mathrm{~g}$ de $\mathrm{MS}^{2}$ & 1205,0 & 411,2 \\
\hline Diâmetro geométrico médio, $\mu \mathrm{m}^{3}$ & 345 & 229 \\
\hline Desvio padrão geométrico ${ }^{3}$ & 2,46 & 2,09 \\
\hline Densidade, $\mathrm{g} / \mathrm{cm}^{3}$ & 0,58 & 0,33 \\
\hline
\end{tabular}

A abertura dos silos foi realizada aos 120 dias pós-ensilagem, foram avaliados o $\mathrm{pH}$ e a Acidez Titulável (AT) seguindo recomendações de Silva \& Queiroz (2002), Índice de Acidez (IA) e Índice de Peróxido (IP) de acordo com a metodologia recomendada por Brasil (2009), caso resultado positivo no IP foi feito $o$ teste de Reação de Kreiss/rancidez. O índice de fungos foi analisado por meio de escala definida pelos autores de 1 a 5 sendo 1 totalmente sem a presença de bolores visíveis e 5 silos totalmente tomado por crescimento de bolores. A atividade água (Aw) foi analisada por meio do equipamento modelo Aw Sprint TH 500.

Após a abertura os minis silos foram mantidos em sala climatizada com temperatura de $20^{\circ} \mathrm{C}$, e foram avaliados o $\mathrm{pH}$ e a temperatura interna dos silos após
24, 48 e 120 horas. Foi considerado como prejuízo para a estabilidade aeróbia a elevação da temperatura em $2^{\circ} \mathrm{C}$ acima da temperatura ambiente e o aumento do $\mathrm{pH}$. Foram avaliados cinco níveis de hidratação $(10 ; 20 ; 30 ; 40$ e $50 \%$ adicionados ao total de peso natural) e cinco relações FAR: FMI (10:0, 9:1, 8:2, $7: 3$ e 6:4) perfazendo um delineamento em esquema fatorial $(5 \times 5)$ e com quatro repetições, totalizando 100 unidades experimentais. Paralelamente, foram produzidos silos referência com a mesma metodologia dos demais silos, no qual o FAR foi ensilado sem adição de água e FMI.

Foram ajustados modelos de superfície de resposta, com o seguinte modelo estatístico: $\mathrm{Y}_{\mathrm{ijk}}=\mu+\mathrm{Ti}+\mathrm{G}_{\mathrm{j}}+\left(\mathrm{T}^{*} \mathrm{G}_{\mathrm{ij}}\right)+$ $\mathrm{e}_{\mathrm{ijk}}$ em que: $\mathrm{Y}_{\mathrm{ijk}}=$ valor observado para a variável k, no grau de hidratação i e na 
Rev. Bras. Saúde Prod. Anim., Salvador, v.17, n.4, p.608-616 out./dez., 2016 http://www.rbspa.ufba.br ISSN 15199940

inclusão de FMI j; $\mu=$ constante geral; $T_{i}$ $=$ efeito do grau de hidratação $(10 ; 20 ; 30$; 40 e $50 \%) ; \mathrm{G}_{\mathrm{j}}=$ efeito do nível de FMI $\mathrm{j}$ $(0 ; 10 ; 20 ; 30$ e $40 \%) ; \mathrm{T}^{*} \mathrm{G}_{\mathrm{ij}}=$ efeito da interação da hidratação i com FMI j e $e_{i j k}$ $=$ erro aleatório associado a cada observação. Foram considerados para a elaboração das equações os modelos que apresentaram significância menor ou igual a 5\%. Posteriormente, a média do grupo controle foi comparada com as médias dos demais tratamentos pelo teste de Dunnett $(\mathrm{P}<0,05)$.

\section{RESULTADOS E DISCUSSÃO}

Houve interação $(\mathrm{P}<0,05)$ do nível de hidratação com o nível de adição de FMI para as variáveis $\mathrm{pH}, \mathrm{AT}$, IA e IF (Tabela 2). Com desdobramento dos resultados, para os níveis de inclusão de FMI, obteve-se efeito $(\mathrm{P}<0,05) \quad$ linear decrescente para as variáveis $\mathrm{pH}$ e AT e IA e IF (Tabela 2). E para os níveis de hidratação houve efeito $(\mathrm{P}<0,05)$ quadrático com ponto de mínimo para $\mathrm{o}$ $\mathrm{pH}$ e quadrático com ponto de máximo para as variáveis AT, IA, IF e Aw. Os valores obtidos para o tratamento referência: pH 6,09; Acidez titulável 3,95; índice de acidez 4,32\%, índice de fungos 0,0 e atividade água 0,59 foram diferentes dos demais tratamentos $(\mathrm{P}<0,05)$ o que indica a efetividade do processo ensilagem, em especial para variáveis como o $\mathrm{pH}$ e menor efetividade para índice de fungos.

Tabela 2. Efeito da ensilagem do farelo de arroz integral (FAR) com níveis crescentes de hidratação e adição de fubá de milho (FMI), no pH, acidez titulável (AT), índice de acidez (IA), índice de fungos (IF) e atividade de água (Aw)

\begin{tabular}{|c|c|c|c|c|c|}
\hline Variáveis & $\mathrm{pH}$ & $\mathrm{AT},{ }^{\circ} \mathrm{D}$ & IA, $\%$ & IF & Aw \\
\hline \multicolumn{6}{|l|}{ Níveis de água, \% } \\
\hline 10 & 5,72 & 09,73 & 17,13 & 1,75 & 0,90 \\
\hline 20 & 5,22 & 24,24 & 22,14 & 2,95 & 0,93 \\
\hline 30 & 4,36 & 37,22 & 16,27 & 3,00 & 0,94 \\
\hline 40 & 4,27 & 36,92 & 12,45 & 1,62 & 0,96 \\
\hline 50 & 4,29 & 35,31 & 12,00 & 1,92 & 0,97 \\
\hline Efeito linear & $<0,01$ & $<0,01$ & 0,16 & $<0,01$ & $<0,01$ \\
\hline Efeito quadrático & $<0,01$ & $<0,01$ & $<0,01$ & $<0,01$ & $<0,01$ \\
\hline \multicolumn{6}{|l|}{ Níveis de fubá, \% } \\
\hline 0 & 4,91 & 30,16 & 18,74 & 2,05 & 0,94 \\
\hline 10 & 4,82 & 29,79 & 17,00 & 1,77 & 0,94 \\
\hline 20 & 4,75 & 29,62 & 16,25 & 2,62 & 0,94 \\
\hline 30 & 4,73 & 27,61 & 14,68 & 2,67 & 0,94 \\
\hline 40 & 4,64 & 26,25 & 13,32 & 2,12 & 0,94 \\
\hline Efeito linear & $<0,01$ & $<0,01$ & $<0,01$ & 0,01 & $\mathrm{NS}$ \\
\hline Efeito quadrático & NS & NS & NS & NS & NS \\
\hline Efeito da Interação & $<0,01$ & $<0,01$ & $<0,05$ & 0,03 & NS \\
\hline $\mathrm{R}^{2}$ & 0,87 & 0,87 & 0,54 & 0,15 & 0,60 \\
\hline \multicolumn{6}{|c|}{$\begin{array}{l}\mathrm{pH}=7,1326-1,61 \mathrm{Mi}-12,1472 \mathrm{H} 2 \mathrm{O}+12,8179 \mathrm{H} 2 \mathrm{O}^{2}+3,2550 \mathrm{Mi} * \mathrm{H} 2 \mathrm{O} . \\
\mathrm{AT}=-17,8841+23,32 \mathrm{Mi}+281,1090 \mathrm{H} 2 \mathrm{O}-325,1070 \mathrm{H}^{2} \mathrm{O}^{2}-111,05 \mathrm{Mi}^{*} \mathrm{H} 2 \mathrm{O} . \\
\mathrm{IA}=21,8795-21,6126 \mathrm{Mi}+12,3927 \mathrm{H} 2 \mathrm{O}-63,3213 \mathrm{H}_{2} \mathrm{O}^{2}+28,1753 \mathrm{Mi} * \mathrm{H} 2 \mathrm{O} . \\
\mathrm{IF}=0,0225+4,5375 \mathrm{Mi}+15,1714 \mathrm{H} 2 \mathrm{O}-23,0357 \mathrm{H} 2 \mathrm{O}^{2}-11,6250 \mathrm{Mi}^{*} \mathrm{H} 2 \mathrm{O} . \\
\mathrm{Aw}=0,88+0,31 \mathrm{H} 2 \mathrm{O}-0,25 \mathrm{H} 20^{2} .\end{array}$} \\
\hline
\end{tabular}


A diminuição do $\mathrm{pH}$ da silagem, para valores próximos a 4,0, auxilia na preservação da massa em virtude da inibição da atividade das bactérias do gênero Clostridium, que são responsáveis pela produção do ácido butírico e pela deterioração da silagem (MOTA et al., 2011). Segundo McDonald et al. (1991) uma silagem de boa qualidade apresenta valores de $\mathrm{pH}$ entre 3,8 e 4,2.

No estudo, a inclusão do FMI apresentou efeito linear $(\mathrm{P}<0,05)$ decrescente sobre $\mathrm{O}$ $\mathrm{pH}$, associado, provavelmente, a maior quantidade de carboidratos solúveis do FMI e por consequência favorecimento do processo fermentativo associado à menor capacidade tampão (Tabela 1).

Por outro lado, a inclusão de água proporcionou efeito quadrático, com melhores resultados para o $\mathrm{pH}$, de acordo com o nível de hidratação entre 42 e $47 \%$ (Tabela 4).

Os tratamentos com 10 e $20 \%$ de inclusão de água não proporcionaram $\mathrm{pH}$ adequado, o que corrobora com os resultados reportados por Goodrich et al. (1975) em que níveis de hidratação inferiores à $18 \%$ prejudicam a fermentação e a qualidade da ensilagem, e das afirmações de Ferrari Junior \& Lavezzo (2001) que o excesso de umidade propicia condições para obtenção de silagens butíricas de baixa qualidade, onde há grande decomposição proteica, com evidente queda no valor nutritivo da massa ensilada.

Tais hipóteses são reforçadas pelo comportamento quadrático com ponto de máximo para a Aw, a derivação da equação indicou ponto de inflexão de $62 \%$, fora do limite estudado (10 e 50\% de hidratação). Provavelmente, os níveis intermediários de Aw tenham maximizado o crescimento microbiano e consequentemente o comportamento quadrático obtido nas demais variáveis. Segundo McDonald et al. (1991), o crescimento de bactérias do gênero
Clostridium é inibido com Aw abaixo de 0,94 , enquanto que as bactérias ácido láticas são menos sensíveis.

Em relação a AT, esta apresentou comportamento inverso ao $\mathrm{pH}$ com efeito $(\mathrm{P}<0,05)$ quadrático com ponto de máximo para o nível de hidratação e linear decrescente com a inclusão de FMI. Apesar da capacidade tampão do FAR ser 5,7 vezes maior que o milho e o $\mathrm{pH}$ inicial ter se mostrado maior no momento da ensilagem.

Autores como Jobim et al. (2007) consideram a AT como o melhor indicador para a avaliação da qualidade da silagem, pois indica a concentração de ácidos orgânicos, especificamente, para silagens com FAR, esta variável pode não estar associada a melhor qualidade. Tal hipótese é baseada no fato da AT estar relacionada à presença de ácidos orgânicos e deve ser ressaltado que tais ácidos podem ser originários da hidrólise dos triglicerídeos do alimento e não necessariamente da atividade microbiana, hipótese é reforçada pelo fato que na silagem com $100 \%$ de FAR os silos possuíam cerca de $22,01 \%$ de extrato etéreo na MS enquanto que no tratamento com $40 \%$ de milho o valor era de $14,94 \%$ de EE na MS. A maior proporção de triglicerídeos pode ter resultado em uma maior hidrolização e maior produção relativa de ácidos graxos livres, portanto a variável $\mathrm{pH}$, neste caso, pode ser um indicativo melhor da qualidade da silagem de FAR.

No tratamento referência a reação de Kreiss/rancidez, foi positiva o que indica a presença de substâncias rançosas, definindo certo grau de oxidação da gordura, enquanto para todos os demais tratamentos não houve resultado positivo na análise, o que sugere eficácia da ensilagem como ferramenta para inibir a peroxidação lipídica e favorecer a conservação do FAR. 
Rev. Bras. Saúde Prod. Anim., Salvador, v.17, n.4, p.608-616 out./dez., 2016 http://www.rbspa.ufba.br ISSN 15199940

As variáveis de estabilidade da silagem (Tabela 3), semelhante à maioria das variáveis de qualidade, apresentaram interação $(\mathrm{P}<0,05)$ entre $\mathrm{o}$ grau de hidratação e inclusão de FMI. As derivações das equações de acordo com o nível de inclusão de FMI resultaram valores específicos de hidratação (Tabela 4).

Tabela 3. Efeito da ensilagem do farelo de arroz integral (FAR) com níveis crescentes de hidratação e adição de fubá de milho (FMI), na estabilidade pós abertura

\begin{tabular}{|c|c|c|c|c|c|c|}
\hline \multirow{2}{*}{ Níveis de Água \% } & \multicolumn{3}{|c|}{$\mathrm{pH}$} & \multicolumn{3}{|c|}{ Temperaturas, ${ }^{\circ} \mathrm{C}$} \\
\hline & $24 \mathrm{~h}$ & $48 \mathrm{~h}$ & $120 \mathrm{~h}$ & $24 \mathrm{~h}$ & $48 \mathrm{~h}$ & $120 \mathrm{~h}$ \\
\hline 10 & 5,70 & 5,82 & 5,81 & 20,40 & 22,38 & 22,98 \\
\hline 20 & 5,18 & 5,27 & 5,22 & 20,03 & 21,16 & 22,76 \\
\hline 30 & 4,34 & 4,42 & 4,55 & 20,40 & 22,00 & 23,35 \\
\hline 40 & 4,23 & 4,48 & 5,30 & 20,74 & 27,57 & 27,97 \\
\hline 50 & 4,22 & 5,02 & 5,94 & 21,39 & 30,88 & 32,22 \\
\hline Efeito linear & $<0,01$ & $<0,01$ & $<0,01$ & 0,01 & $<0,01$ & $<0,01$ \\
\hline Efeito quadrático & $<0,01$ & $<0,01$ & $<0,01$ & $<0,01$ & $<0,01$ & $<0,01$ \\
\hline \multicolumn{7}{|l|}{ Níveis de fubá \% } \\
\hline 0 & 4,84 & 4,92 & 4,96 & 20,51 & 21,74 & 22,91 \\
\hline 10 & 4,77 & 5,01 & 5,36 & 20,48 & 24,39 & 24,70 \\
\hline 20 & 4,72 & 5,07 & 5,48 & 20,81 & 26,17 & 28,02 \\
\hline 30 & 4,70 & 5,05 & 5,52 & 20,52 & 26,38 & 26,76 \\
\hline 40 & 4,65 & 4,97 & 5,50 & 20,64 & 25,32 & 26,88 \\
\hline Efeito linear & $<0,01$ & $<0,01$ & $<0,01$ & 0,05 & $<0,01$ & 0,07 \\
\hline Efeito quadrático & NS & NS & NS & NS & NS & NS \\
\hline Efeito da interação & 0,02 & $<0,01$ & $<0,01$ & 0,02 & $<0,01$ & $<0,01$ \\
\hline $\mathrm{R}^{2}$ & 0,89 & 0,69 & 0,61 & 0,24 & 0,69 & 0,58 \\
\hline $\begin{array}{l}\mathrm{pH} 24 \mathrm{~h}=7,0215-1,1 \\
\mathrm{pH} 48 \mathrm{~h}=7,6780-2,0 \\
\mathrm{pH} 120 \mathrm{~h}=7,6291-2, \\
\text { Temperatura } 24 \mathrm{~h}=2 \\
\text { Temperatura } 48 \mathrm{~h}=2 \\
\text { Temperatura } 120 \mathrm{~h}=\end{array}$ & $\begin{array}{l}1,9504 \mathrm{H} \\
7,0821 \mathrm{H} \\
18,5472 \\
, 3775 \mathrm{M} \\
1,0999 \mathrm{~N}\end{array}$ & $\begin{array}{l}2,6464 \mathrm{H} \\
2,0285 \mathrm{H} \\
27,7178 \\
93 \mathrm{H} 2 \mathrm{O} \\
1572 \mathrm{H} 2\end{array}$ & $\begin{array}{l}+2,27 \mathrm{M} \\
+7,36 \mathrm{M} \\
2+11,35 \\
571 \mathrm{H} 2 \mathrm{C} \\
3,4288 \mathrm{H}\end{array}$ & $\begin{array}{l}\text { \% } \\
{ }^{*} \mathrm{H} 2 \mathrm{O} . \\
250 \mathrm{Mi}^{*} \\
67,4998\end{array}$ & 20. & \\
\hline
\end{tabular}

Houve efeito linear decrescente $(\mathrm{P}<0,05)$ com o aumento da inclusão de FMI, para o pH após 24 horas de abertura, de forma que a inclusão de $40 \%$ foi a mais adequada. No referido nível de inclusão de FMI (40\%) o nível de água para maximizar a acidificação 24 horas pós abertura foi de $43,7 \%$ do peso (Tabela 4). Considerando a equação: \% de água final $=\{100 *[\%$ de água adicionada sob a massa $+(\%$ de $\mathrm{FAR} * \%$ de água do FAR $+\%$ de FMI * $\%$ de água no $\mathrm{FMI}] /[\%$ de água adicionada sob a massa+ (\% de FAR + $\%$ de FMI)]\}, a umidade total, nos níveis recomendados para minimizar o pH após 24 horas da abertura do silo, foi estimada em aproximadamente $38,5 \%$, este valor é próximo às recomendações de Pereira (2012) que avaliou diferentes graus de reidratação de milho e recomendou níveis de umidade entre 35 e $38 \%$ para a obter o $\mathrm{pH}$ mais adequado das silagens, mas acima dos valores recomendados por McDonald et al. (1991) de 30 a 35\% de umidade.

A partir das 48 horas de abertura houve efeito $(\mathrm{P}<0,05)$ linear crescente com a inclusão do FMI para o pH (Tabela 3), 
Rev. Bras. Saúde Prod. Anim., Salvador, v.17, n.4, p.608-616 out./dez., 2016 http://www.rbspa.ufba.br

resultados inversos ao obtido para a variável pH 24 horas a qual apresentou decréscimo com a adição do FMI. O aumento do $\mathrm{pH}$, após 24 horas de abertura com a inclusão do FMI, provavelmente está associado a maior disponibilidade de carboidratos solúveis do FMI os quais, após o benefício inicial de maior acidificação, favorecem o desenvolvimento de microrganismos aeróbicos e com isso o aumento do $\mathrm{pH}$ e da temperatura da silagem. A deterioração das silagens é geralmente manifestada pelo aumento na temperatura, mas variável entre os tipos de forragens ensiladas (CASTRO et al., 2006).

Tabela 4. Nível ideal de hidratação obtidos pelas derivações das equações em função da inclusão do fubá de milho (FMI) para as variáveis de Acidez titulável (AT) pH e temperatura após abertura do silo de farelo de arroz integral (FAR)

\begin{tabular}{|c|c|c|c|c|c|c|c|c|}
\hline \multirow{2}{*}{ FMI, \% } & \multirow[b]{2}{*}{ AT } & \multicolumn{4}{|c|}{$\mathrm{pH}$} & \multicolumn{3}{|c|}{ Temperaturas, ${ }^{\circ} \mathrm{C}$} \\
\hline & & $\mathrm{Oh}$ & $24 \mathrm{~h}$ & $48 \mathrm{~h}$ & $120 \mathrm{~h}$ & $24 \mathrm{~h}$ & $48 \mathrm{~h}$ & $120 \mathrm{~h}$ \\
\hline 0 & 43,2 & 47,4 & 47,3 & 38,8 & 33,5 & 26,9 & 25,0 & 24,0 \\
\hline 10 & 41,5 & 46,1 & 46,4 & 37,1 & 31,4 & 23,8 & 21,5 & 20,6 \\
\hline 20 & 39,8 & 44,8 & 45,5 & 35,4 & 29,4 & 20,7 & 18,1 & 17,2 \\
\hline 30 & 38,1 & 43,6 & 44,6 & 33,8 & 27,3 & 17,6 & 14,7 & 13,8 \\
\hline 40 & 36,4 & 42,3 & 43,7 & 32,1 & 25,3 & 14,5 & 11,3 & 10,4 \\
\hline
\end{tabular}

Valores estimados com base nas equações obtidas (Tabela 2 e 3). AT= acidez titulável.

A ensilagem inibiu a peroxidação das gorduras e se mostrou promissora como ferramenta para aumentar o tempo de conservação do FAR, visto não ser detectada peroxidação em nenhum dos tratamentos. Conquanto os resultados sejam promissores com melhora na qualidade e na conservação do FAR outros trabalhos devem ser conduzidos para avaliar os efeitos da ensilagem sobre o desempenho animal, viabilidade econômica, nível de micotoxinas, entre outros.

A inclusão de $40 \%$ de FMI melhorou o $\mathrm{pH}$ na abertura e com 24 horas pós abertura. O nível de inclusão de $40 \%$ de FMI e 42,3 ou $43,7 \%$ de água foi a mais adequada pois melhorou o $\mathrm{pH}$ da silagem na abertura e com 24 horas, respectivamente. Paralelamente, aos benefícios com a maior acidificação com a inclusão do milho, o aumento do volume da massa, permitirá maior corte do perfil do silo.
A análise do $\mathrm{pH}$ indicou que as silagens estavam deterioradas a partir das 24 horas de abertura e a inclusão do milho acelerou a deterioração da massa ensilada após este período.

Para a confecção de silagem de farelo de arroz recomenda-se a inclusão de FMI de $40 \%$ e hidratação de $42,3 \%$ sobre o peso para melhorar o $\mathrm{pH}$ na abertura.

\section{REFERÊNCIAS}

ALI, M.M.; HUSSAIN, M.G.; NURUL, A.B.S.A.R.; SHAHJAHAN. M.;

ABSAR, N. Investigation on rice bran: composition of rice bran and its oil.

Bangladesh Journal of Scientific and Industrial Research, v.33, n.2, p.170177, 1998. 
Rev. Bras. Saúde Prod. Anim., Salvador, v.17, n.4, p.608-616 out./dez., 2016 http://www.rbspa.ufba.br ISSN 15199940

ÁVILA, C.L.S.; PINTO, J.C.; EVANGELISTA, A.R.; MORAIS, A.R.; FIGUEIREDO, H.C.P.; TAVARES, V.B. Perfil de fermentação das silagens de capim-tanzânia com aditivos: teores de nitrogênio amoniacal e $\mathrm{pH}$. Ciência e Agrotecnologia, v.27, n.5, p.1144-1151, 2003.

BRASIL. Ministério da Agricultura e Abastecimento. Sindicato Nacional de Indústria de Alimentação Animal. Associação Nacional dos Fabricantes de Rações. Compêndio brasileiro de alimentação animal. São Paulo: ANFAR/CBNA/SDR, 2009.

CASTRO, F.G.F.; NUSSIO, L.G.; HADDAD, C.M.; CAMPOS, F.P.; COELHO, R.M.; MARI, L.J.; TOLEDO, P.A.; Perfil microbiológico, parâmetros físicos e estabilidade aeróbia de silagens de capim-tifton 85 (Cynodon ssp.) confeccionadas com distintas concentrações de matéria seca e aplicação de aditivos. Revista Brasileira de Zootecnia, v.35, n.2, p.358-371, 2006.

COMPANHIA NACIONAL DE ABASTECIMENTO - CONAB. Acompanhamento da safra brasileira de grãos. Safra 2014/2015. Brasília, v.2, n.9, p.1-104, 2015.

CORDERO, V.; BARBER, C.B.; CLEMENTE, G.; PRIMO, E.

Inactivación enzimática del salvado de arroz. Relacion entre los parámetros del processo y la actividad de peroxidasa. Revista Agroquimica y Tecnologiade Alimentos, v.25, n.1, p.75-86, 1985.

FERRARI JUNIOR, E.; LAVEZZO, W. Qualidade da silagem de capim-elefante (Pennisetum purpureum Schum) e murchecido ou acrescido de farelo de mandioca. Revista Brasileira de Zootecnia, v.30, n.5, p.1424-1431, 2001.
GOODRICH, R.D.; BYERS, F.M.; MEISKE, J.C. Influence of Moisture Content, Processing and Reconstitution on the Fermentation of Corn Grain. Journal of Animal Science, v.41, n.3, p.876-881, 1975.

HANDERSON, S.M.; PERRY, R.L. Agricultural process engineering. New York: John Wiley and Sons, 1955. 402 p.

JOBIM, C.C.; NUSSIO, L.G.; REIS, R.A.; SCHMIDT, P. Avanços metodológicos na avaliação da qualidade da forragem conservada. Revista Brasileira de Zootecnia, v.36, p.101119, 2007. Suplemento especial.

KAUFMANN, C.; SAUER, W.C.; CERVANTES, M.; ZHANG, Y; HE, J.; RADEMACHER, M.; HTOO, J.K. Amino acid and energy digestibility in different sources of rice bran for growing pigs. Canadian Journal of Animal Science, v.85, n.3, p.355-363, 2005.

McDONALD, P.; HENDERSON, A.R.; HERON, S.J.E. The biochemistry of silage. 2.ed. Marlow. Chalcombe Publications, 1991. 226p.

MOTA, A.D.S.; ROCHA JÚNIOR, V.R.; SOUZA, A.S.; REIS, S.T.; TOMICH, T.R.; CALDEIRA, L.A.; MENEZES, G.C.C.; COSTA, M.D. Perfil de fermentação e perdas na ensilagem de diferentes frações da parte aérea de quatro variedades de mandioca. Revista Brasileira de Zootecnia, v.40, n.7, p.1466-1473, 2011.

MUJAHID, A.; ASIF, M.; UL HAQ, I.; ABDULLAH, M.; GILANI, A.H. Nutrient digestibility of broiler feeds containing different levels of variously processed rice bran stored for different periods. Poultry Science, v.82, n.9, p.1438-1443, 2003. 
Rev. Bras. Saúde Prod. Anim., Salvador, v.17, n.4, p.608-616 out./dez., 2016 http://www.rbspa.ufba.br

NEUMANN, M.; MÜHLBACH, P.R.F.; NÖRNBERG, J.L.; OST, P.R.; RESTLE, J.; SANDINI, I.E.; ROMANO, M.A.

Características da fermentação da silagem obtida em diferentes tipos de silos sob efeito do tamanho de partícula e da altura de colheita das plantas de milho. Ciência Rural, v.37, n.3, p.847-854, 2007.

PEREIRA, M.N. Milho rehidratado e ensilado na alimentação de vacas leiteiras.

Jornal Holândes, p.22-23, 2012.

PLAYNE, M.J.; McDONALD, P. The buffering constituents of herbage and of silage. Journal of the Science of Food and Agriculture, v.17, n.6, p.264-268, 1966.

ROSTAGNO, H.S.; ALBINO, L.F.T.;

DONZELE, J.L.; GOMES, P.C.;

OLIVEIRA, R.F.; LOPES, D.C.;

FERREIRA, A.S.; BARRETO, S.L.T.; EUCLIDES, R.F. Tabelas brasileiras

para aves e suínos: composição de alimentos e exigências nutricionais. 3.ed. Viçosa, MG: UFV, 2011. 252p.

SAUNDERS, R.M. The properties of rice bran as a foodstuff. Cereals Foods World, v.35, n.7, p.632-636, 1990.

SILVA, D.J.; QUEIROZ, A.C. Análise de alimentos: métodos químicos e biológicos. 2.ed. Viçosa: UFV, 2002. $235 \mathrm{p}$.

WEINBERG, Z.G.; CHEN, Y. Effects of storage period on the composition of whole crop wheat and corn silages.

Animal Feed Science and Technology, v.185, n.3, p.196-200, 2013.

Data de recebimento: 02/10/2015

Data de aprovação: 15/09/2016 